\title{
Crecimiento de langosta espinosa (Panulirus argus) en jaulas flotantes en el Refugio de Vida Silvestre Cayos Perla, municipio de Laguna de Perlas, RACCS, Nicaragua
}

\author{
Growth of spiny lobster in floating cages in Cayos Perla Wildlife Refuge, Laguna de Perlas municipality, \\ RACCS, Nicaragua
}

\author{
Eduardo Siu Estrada' ${ }^{1}$ \\ Lindolfo Hodgson Suárez ${ }^{2}$ \\ Juan Suárez Sánchez ${ }^{3}$ \\ Hans van der Wal ${ }^{4}$ \\ Darven Siu Estrada ${ }^{5}$ \\ Jasper Romero Ebanks ${ }^{6}$
}

\section{Resumen}

La langosta común (Panulirus argus) es una especie con alto valor comercial en el Caribe nicaragüense. En las últimas décadas su producción ha alcanzado sus valores máximos, aunado a las fluctuaciones asociadas a la variabilidad climática y su mismo aprovechamiento, demandan la necesidad de incursionar en formas complementarias de producción, como lo es la acuicultura. En el Refugio de Vida Silvestre Cayos Perla, Nicaragua, se evaluó el crecimiento de 85 langostas (P. argus), cultivadas en una jaula flotante de 2 x $2 \times$ $2.30 \mathrm{~m}$, durante el período del 21 de diciembre de 2018 al 23 de mayo de 2019, alimentadas diariamente con sardinas a razón del $7 \%$ de su peso.

Durante este período el agua presentó valores promedios de temperatura de $28.5^{\circ} \mathrm{C}$, $\mathrm{pH}$ de 7.6 , salinidad de $34.4 \mathrm{~g} \mathrm{~L}^{-1}$, turbidez mayor a un metro y velocidad de la corriente de 0.2 a $0.4 \mathrm{ft} \mathrm{s}^{-1}$. Se observaron dos etapas de crecimiento de la langosta, de 89 y 64 días, cada una.

La primera caracterizada por nulo crecimiento, densidad de 10.6 organismos $\mathrm{m}^{3}$ y mortalidad alta al final (39\%); la segunda por un crecimiento similar al observado en su estado natural, sobrevivencia alta $(100 \%)$, con una densidad de 6.3 organismos $\mathrm{m}^{3}$.

La relación peso (W) - longitud del cefalotórax (LC) de las langostas durante el cultivo se ajustó a la ecuación $\mathrm{W}=0.02788$ LC 2.2074. De lo anterior se puede decir que el cultivo de la langosta en ésta región tiene un alto potencial.

Palabras clave: Acuicultura; Caribe; Panulirus argus.

\footnotetext{
1 MSc. en Biología Marina; Decano Facultad de Recursos Naturales y Medio Ambiente Bluefields Indian \& Caribbean University (BICU).No ORCID: http://orcid. org/0000-0002-5104-747X; correo: eduardosiue@yahoo.com

2 Ingeniero Acuícola, Técnico del proyecto ecosistemas costeros, Instituto de Biodiversidad y Estudios Ambientales (IBEA). Facultad de Recursos Naturales y Medio Ambiente. Bluefields Indian \& Caribbean University (BICU).

3 PhD. Director Laboratorio de Recursos Naturales Facultad de Agrobiología universidad Autónoma de Tlaxcala, ORCID: http://orcid.org/0000-0002-9127-7413

4 PhD. Director Departamento de Agricultura, Sociedad y Ambiente. El Colegio de la Frontera Sur, Villahermosa. ORCID: http://orcid.org/0000-0002-2765-8596

5 Ing agroforestal. Coordinador del proyecto Ecosistemas costeros en Bluefields Indian \& Caribbean University (BICU). Correo: darrendanny@gmail.com

6 Master en Gestión Sostenible de Recursos Naturales. Director del Instituto de Biodiversidad y Estudios Ambientales (IBEA), Bluefields Indian \& Caribbean University (BICU)
}

Recibido: 08/08/2019 - Aprobado: 30/08/2019 


\section{Abstract}

The common lobster (Panulirus argus) is a species with high commercial value in the Nicaraguan Caribbean. In recent decades its production has reached its maximum values, coupled with the fluctuations associated with climate variability and its use, demand the need to enter into complementary forms of production, such as aquaculture. In the Cayos Perla Wildlife Refuge, Nicaragua, the growth of 85 lobsters (P. argus), grown in a floating cage of $2 \times 2 \times 2.30 \mathrm{~m}$, was evaluated during the period from December 21, 2018 to May 23 of 2019 , fed daily with sardines at a rate of $7 \%$ of its weight.

During this period the water presented average temperature values of $28.5^{\circ} \mathrm{C}, \mathrm{pH} 7.6$, salinity of $34.4 \mathrm{~g}$ $\mathrm{L}^{-1}$, turbidity greater than one meter and current velocity of 0.2 to $0.4 \mathrm{ft} \mathrm{s}^{-1}$. Two stages of lobster growth were observed, 89 and 64 days, each.

The first characterized by zero growth, density of 10.6 organisms $\mathrm{m}^{3}$ and high mortality at the end (39\%); the second by a growth similar to that observed in its natural state, high survival (100\%), with a density of $6.3 \mathrm{~m}^{3}$ organisms.

The weight $(\mathrm{W})$ - cephalothorax $(\mathrm{LC})$ ratio of lobsters during cultivation was adjusted to equation $\mathrm{W}=$ 0.02788 LC 2.2074. From the above it can be said that lobster cultivation in this region has a high potential.

Keywords: Aquaculture; Caribbean; Panulirus argus.

\section{Introducción}

La langosta (Panulirus argus) es una especie con alto valor comercial en el Atlántico centro occidental, 26 países están involucrados en su aprovechamiento y comercialización; los principales países productores con más del 6o\% del total de las capturas son Cuba, Bahamas y Brasil, seguidos por Estados Unidos, Honduras y Nicaragua (Ehrhardt, 2005; Alzugaray Martínez \& Puga Millán, 2012).

La pesquería de la langosta ( $P$. argus) en el Caribe nicaragüense representa el rubro económico del sector pesquero más importante del país, ya que es una considerable fuente de empleo para los grupos sociales más deprimidos de la región (FAO, 2003).

La producción total de langosta (P. argus) en las últimas décadas, muestra una estabilidad y la demanda por este recurso se sigue incrementando. Estas condiciones, aunadas a las características propias de la langosta, gregarismo, tolerancia a rangos amplios de condiciones ambientales, aceptable crecimiento, relativamente pocas enfermedades y aceptación de una amplia variedad de alimento natural; hacen a esta especie susceptible para la acuicultura (Jeffs, 2010).

En la región del Refugio de Vida Silvestre Cayos Perla, los productores de langosta, demandan alternativas para enfrentar problemas como la disminución de las capturas debido a mayores esfuerzos pesqueros y captura de ejemplares fuera de talla, así como la variabilidad en las capturas de un año a otro. Por lo que el cultivo de la langosta puede ser una opción que contribuya a incrementar la producción de este valioso recurso.

Por lo anterior, el objetivo de este trabajo es evaluar el crecimiento de la langosta espinosa (Panulirus argus) en jaulas flotantes en el Refugio de Vida silvestre Cayos Perla, municipio de Laguna de Perlas, R.A.C.C.S., con el uso de modelos de crecimiento, para generar información útil en la gestión sustentable de este recurso. 


\section{Revisión de literatura}

Ríos Lara y otros (1998), realizaron una prospección en el área central del arrecife Alacranes (México), con el propósito de evaluar la abundancia de la langosta (Panulirus argus); para la estimación de la abundancia realizaron un muestreo aleatorio irrestricto, considerando un área de $171495200 \mathrm{~m}^{2}$, entre las coordenadas $22^{\circ} 22^{\prime}-22^{\circ} 35^{\prime}$ de latitud N y $89^{\circ} 37^{\prime}-89^{\circ} 47^{\prime}$ de longitud W, bajo el supuesto de $80 \%$ de área habitable por la langosta. Observaron una densidad promedio de 3.31 organismos ha ${ }^{-1}$, con una abundacia total de 45435 langostas (IC \pm 302 ).

Jeffs y Davis (2003), reportaron que la fuerte demanda de langosta espinosa en el mercado internacional, genera el interés en el desarrollo del cultivo de ésta especie, por lo cual revisaron la información de Panulirus argus de Florida y el Caribe; concluyen que las perspectivas para el cultivo de la langosta espinosa es más rentable que las especies de regiones templadas debido a la gran disponibilidad de larvas silvestres y tasas de crecimiento más rápidas; es factible su cultivo en estanques terrestres y jaulas flotantes, aunque con mayor ganacia económica en jaulas; por lo tanto es necesaria mayor investigación para identificar los sistemas más eficientes; así también investigaciones para establecer densidades y métodos de alimentación.

De León Gónzalez (2005), evalúa los parámetros de crecimiento de la langosta (Panulirus argus) con el modelo de Von Bertalanffy, utilizando el método de NSLCA, con datos de monitoreo biológico del Golfo de Batabanó, Cuba, para el período de 1963 a 2002, encontrando valores promedios para k de 0.2405 año ${ }^{-1}$ (rango: $0.20-0.27$ año $^{-1}$ ), para $L_{\infty}$ de $183.3 \mathrm{~mm} L C\left(170-199.1 \mathrm{~mm}\right.$ ) y $t_{o}$ de 0.45 .

Los parámetros del modelo de Von Bertalanffy para el crecimiento de la Langosta validados para la región del Caribe de Colombia son $\mathrm{k}=0.236, L_{\infty}=186 \mathrm{~mm}$ de $L C$ y $t_{o}=-0.13$ (Borda \& Cruz, 2006).

Solanki y otros (2012), experimentaron diferentes densidades de siembra, 5, 10 y 15 organismo por $\mathrm{m}^{3}$, para el cultivo de langosta (Panulirus polyphagus), en jaulas flotantes, en la bahía de Sarkeshware, India; reportaron tasas de crecimiento específico (SGR), de 0.38, 0.35 y 0.31, para densidades de siembra de 10, 15y 5 , organismos por $\mathrm{m}^{3}$, respectivamente; con sobrevivencias de $24.66,21 \mathrm{y} 0 \%$, para densidades de siembra de 15, 10 y 5 organismo por $\mathrm{m}^{3}$, respectivamente, asociando la sobrevivencia tan baja a las temperaturas bajas del agua durante la estación de invierno.

Matthews y Maxwell (2007), realizaron una serie de experimentos en laboratorio sobre la crianza de la langosta (Panulirus argus) en Cayos Florida, para evaluar el crecimiento y sobrevivencia. La mortalidad observada en estos experimentos fue atribuída principalmente a tres factores: el virus $\mathrm{PaV}_{1}$, pobre nutrición y sobrepoblamiento. Al incio de los experimentos las infecciones virales fueron altamente contagiosas y causaron el 98\% de mortalidad en langostas juveniles; posteriormente de casi eliminar la infección viral, la mortalidad fue asociada a pobre nutrición y sobrepoblamiento, usualmente ocurrió durante la muda, resultando en canibalismo de post muda. Esas fuentes de mortalidad fueron reducidas, utilizando una dieta de moluscos y crustáceos vivos una vez por semana y reduciendo la densidad de las langostas a medida que incrementan su tamaño.

\section{Materiales y métodos}

El Refugio de Vida Silvestre Cayos Perlas, se ubica en la plataforma continental de Nicaragua en el Mar Caribe, frente al litoral marino de la Laguna de Perlas, entre Punta Perlas y las comunidades de Set Net Point y Tasbapauni, en una superficie marina insular de 32000 ha aproximadamente, entre las coordena-

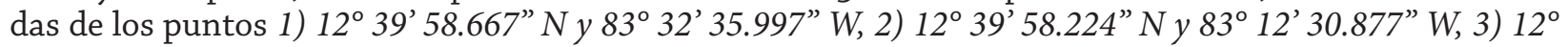

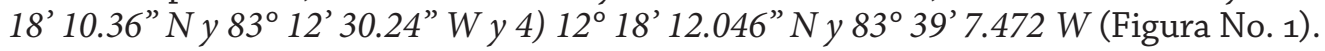




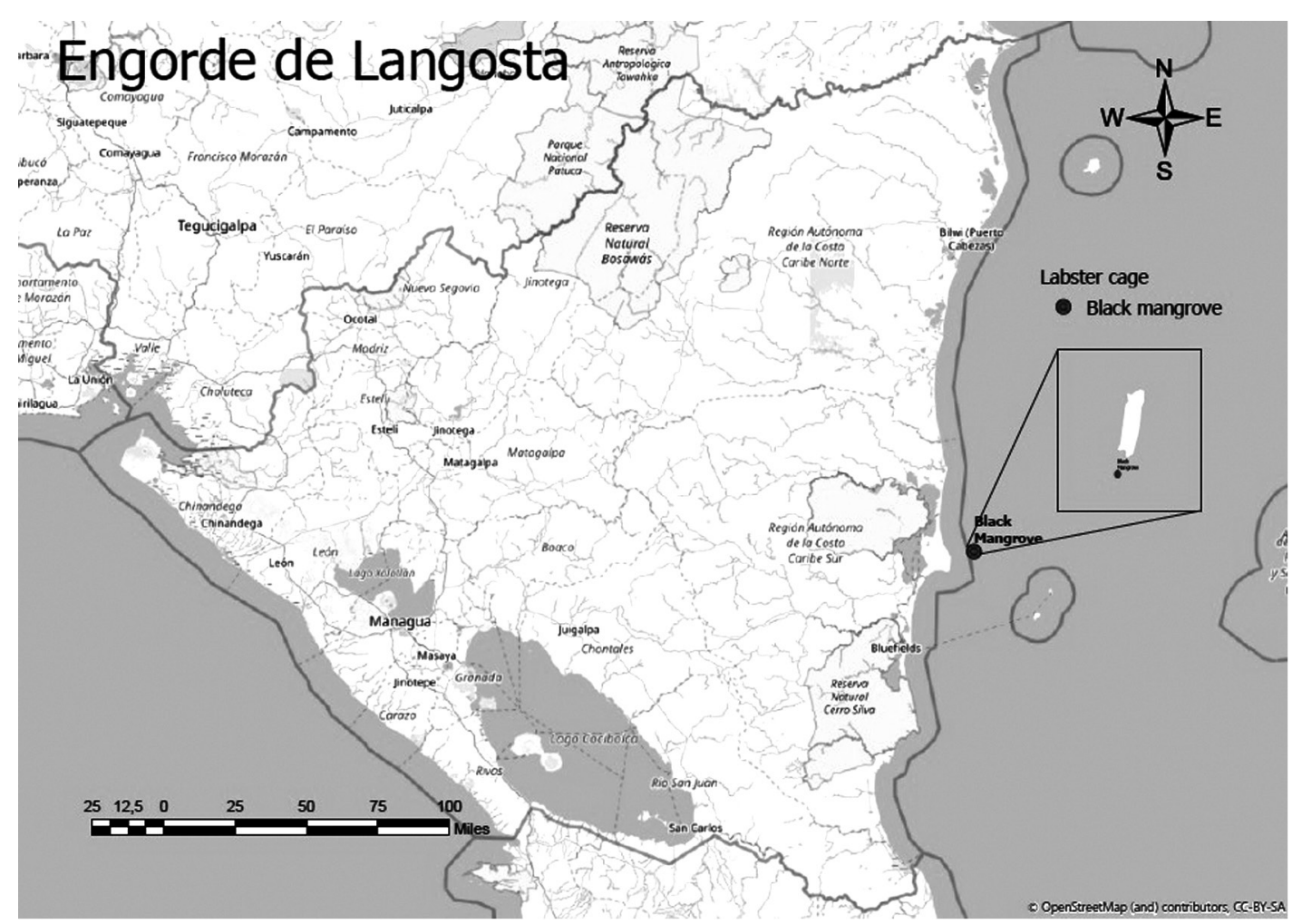

Figura No. 1. Ubicación del Refugio de Vida Silvestre Laguna de Perlas, Nicaragua y la jaula de cultivo de langosta (Panulirus argus).

El 21 de diciembre de 2018, se obtuvieron langostas juveniles y sub-adultas en el monitoreo de refugios artificiales (casitas cubanas) establecidos para el proyecto. Se transportaron en cajas de plástico con capacidad de $100 \mathrm{lb}$ y se colocaron 85 organismos en una jaula flotante de 2 x 2 x $2.30 \mathrm{~m}$ en Black Mangrove Cay en las coordenadas $12.584968^{\circ}$ y $83.311545^{\circ} \mathrm{W}$ (Figura No. 1). Se alimentaron diariamente con sardina (Opisthonema oglinum) a razón del $7 \%$ de su peso, durante 153 días. Se les realizaron biometrías cada 30 días aproximadamente, tomando una muestra aleatoria de 20 organismos y registrando las variables peso (W), longitud total (LT) y longitud del cefalotórax (LC). Se registraron las características del agua, temperatura $\left({ }^{\circ} \mathrm{C}\right), \mathrm{pH}$, salinidad $\left(\mathrm{g} \mathrm{L}^{-1}\right)$, turbidez $(\mathrm{m})$ y velocidad de la corriente $\left(\mathrm{ft} \mathrm{s}^{-1}\right)$, durante el periodo del estudio.

Las jaulas se limpiaron con cepillos de mano y de escoba, cada vez que fue necesario, para evitar y eliminar el acúmulo indeseable de microorganismos, plantas, algas y/o animales en las estructuras, Dentro de las jaulas se colocaron trozos de mallas como medio de protección donde las langostas se pudieran refugiar durante la etapa de la muda y de esta manera evitar el canibalismo.

Para identificar patrones de comportamiento en las variables de crecimiento (W, LC y LT), se realizó un análisis de varianza de una vía (ANOVA), con el software Past, bajo la consideración de violación del supuesto de independencia de dicha metodología.

Para evaluar el crecimiento de la langosta durante el periodo de estudio, se calculó la tasa de crecimiento específico (SGR) con la ecuación (Rivera Menéses, y otros, 2013):

$$
S G R=100\left(\operatorname{Ln} W_{f}-\operatorname{Ln} W_{i}\right) / t
$$

Ec. 1

Donde $W_{f}$ y $W_{i}$ son el peso promedio (g), de las langostas al final y al inicio del estudio, respectivamente y $\mathrm{t}$ es el tiempo en días.

Se utilizaron los ajustes del modelo de Von Bertalanffy al crecimiento de la langosta (Ec. 2), reportados por de Borda \& Cruz, 2006 y de León Gónzalez, 2005, para comparar el crecimiento de la langosta en estado natural con el observado en este estudio. 


$$
L_{t}=L_{\infty}\left\{1-\exp \left[-k\left(t-t_{0}\right)\right]\right\} \quad \text { Ec. } 2
$$

Donde $L_{t}$ es la LC de la langosta en mm al tiempo t (años). $L_{\infty}$ es la LC asintótica promedio (mm), $\mathrm{k}$ es el coeficiente de crecimiento que determina que tan rápido es alcanzado $L_{\infty}$ y $t_{o}$ es la edad hipotética cuando $L_{t}$ es o.

Se ajustó la ecuación de la relación peso - longitud (LC) de la langosta, con los datos registrados en las biometrías y se comparó a los reportados por Borda \& Cruz, 2006 y Alzugaray Martínez \& Puga Millán, 2012.

$$
W=a L C^{b} \quad \text { Ec. } 3
$$

Donde $W$ es el peso de la langosta ( $\mathrm{g}), L C$ es la longitud cefalotórax en $\mathrm{mm}$, a y b, son coeficientes de ajuste lineal.

\section{Resultados y discusión}

Los valores iniciales en el cultivo de la langosta cultivada en jaulas flotantes en el Refugio de Vida Silvestre Cayos Perla, Nicaragua, fueron, 85 organismos con una longitud promedio del cefalotórax de 75.7 $\mathrm{mm}$ (CV de 4.8) y distribución normal (prueba de normalidad Shapiro-Wilk, $\mathrm{p}=0.64$ ), con valor mínimo de $66.5 \mathrm{~mm}$ y máximo de $84 \mathrm{~mm}$ y un solo organismo por encima del límite de la talla comercial (>83 $\mathrm{mm}$ ); una longitud total promedio de $205.2 \mathrm{~mm}$, con valor mínimo de $178 \mathrm{~mm}$ y Máximo de $226 \mathrm{~mm}$; un peso promedio de $386.6 \mathrm{~g}$, con valor mínimo de $296.8 \mathrm{~g}$ y máximo de $451.5 \mathrm{~g}(\mathrm{n}=39)$.

Después de 153 días de cultivo, sobrevivieron 50 organismos (Figura No. 5), con una longitud promedio del cefalotórax de 81.8 ( $\mathrm{n}=20, \mathrm{CV}$ de 6.9), valor mínimo de $70 \mathrm{~mm}$ y máximo de $93 \mathrm{~mm}$, con un $40 \%$ de los organismos con tallas superiores al límite comercial (>83 mm); una longitud total promedio de $223.5 \mathrm{~mm}$ ( $\mathrm{n}=20$ ), con valor mínimo de $200 \mathrm{~mm}$ y máximo de $250 \mathrm{~mm}$; con un peso promedio de $479.6 \mathrm{~g}(\mathrm{n}=20)$, valor mínimo de 330 g y máximo de $672.3 \mathrm{~g}$.

Durante este período se observaron dos etapas de crecimiento diferenciadas estadísticamente en el peso de la langosta (ANOVA, p < 0.01), de o a 69 días y de 70 a 153 días, evidenciadas por la prueba de ManWhitney para pares de valores $\mathrm{p}$, con el método de Bonferroni para valores p corregidos (Figura No. 2); la longitud del cefalotórax (LC) (Figura No. 3), manifestó un incremento de $6.1 \mathrm{~mm}$ para el mismo periodo (Figura No. 3) y la longitud total (LT) un incremento de $18.3 \mathrm{~mm}$ (Figura No. 4).

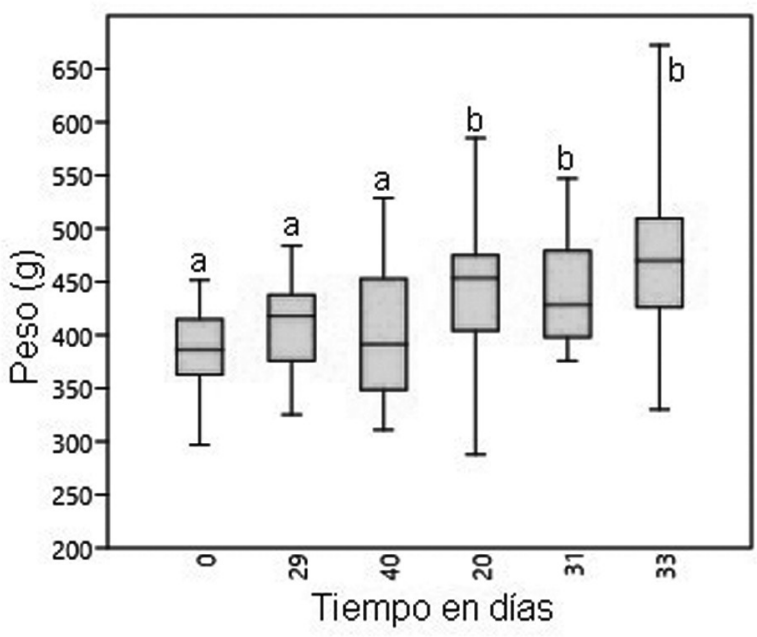

Figura No. 2. Peso de la langosta (Panulirus argus), cultivada en jaulas flotantes en el Refugio de Vida Silvestre Cayos Perla, Nicaragua, del 21 de diciembre de 2018 al 23 de mayo de 2019. a y b son grupos estadísticamente diferentes $p<0.01$. 


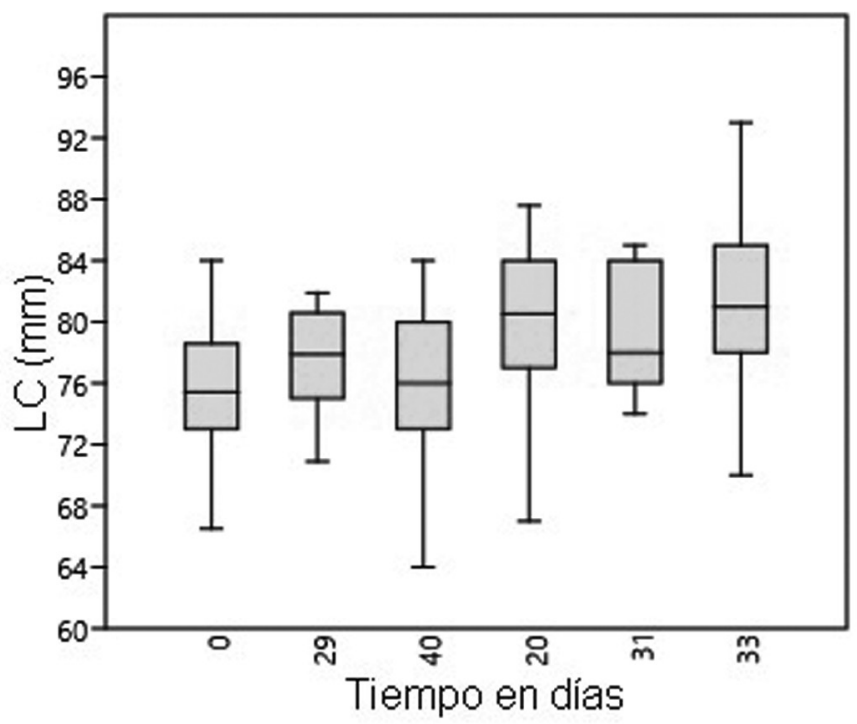

Figura No. 3. Longitud del cefalotórax (LC), de la langosta (Panulirus argus), cultivada en jaulas flotantes en el Refugio de Vida Silvestre Cayos Perla, Nicaragua, del 21 de diciembre de 2018 al 23 de mayo de 2019.

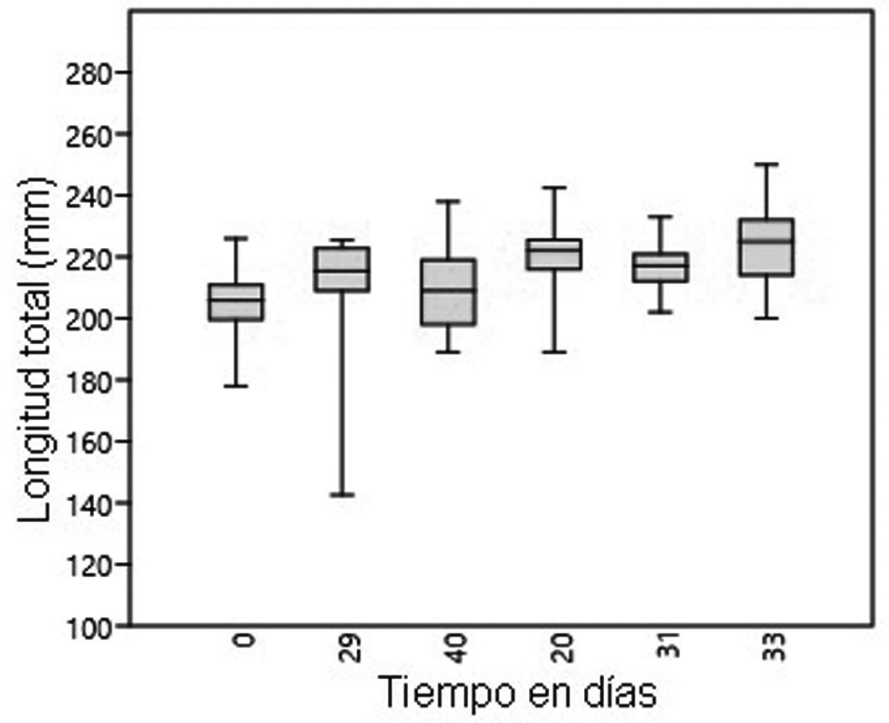

Figura No. 4. Longitud total de la langosta (Panulirus argus), cultivada en jaulas flotantes en el Refugio de Vida Silvestre Cayos Perla, Nicaragua, del 21 de diciembre de 2018 al 23 de mayo de 2019.

La tasa de crecimiento específico (SGR) de la langosta durante el período del estudio, manifestó valores negativos, asociado a los periodos de muda y alta mortalidad (69 y 120 días, respectivamente), así como valores de 0.18 a $0.6 \%$ día $^{-1}$ en las mediciones restantes (Figura No. 5). Los valores de la SGR reportados por Solanki y otros (2012), para el cultivo de la langosta (Panulirus polyphagus), en jaulas flotantes, en la bahía de Sarkeshware, India, de 0.31 a $0.38 \%$ día $^{-1}$, están dentro del rango observado en los valores positivos de la SGR de este estudio. 


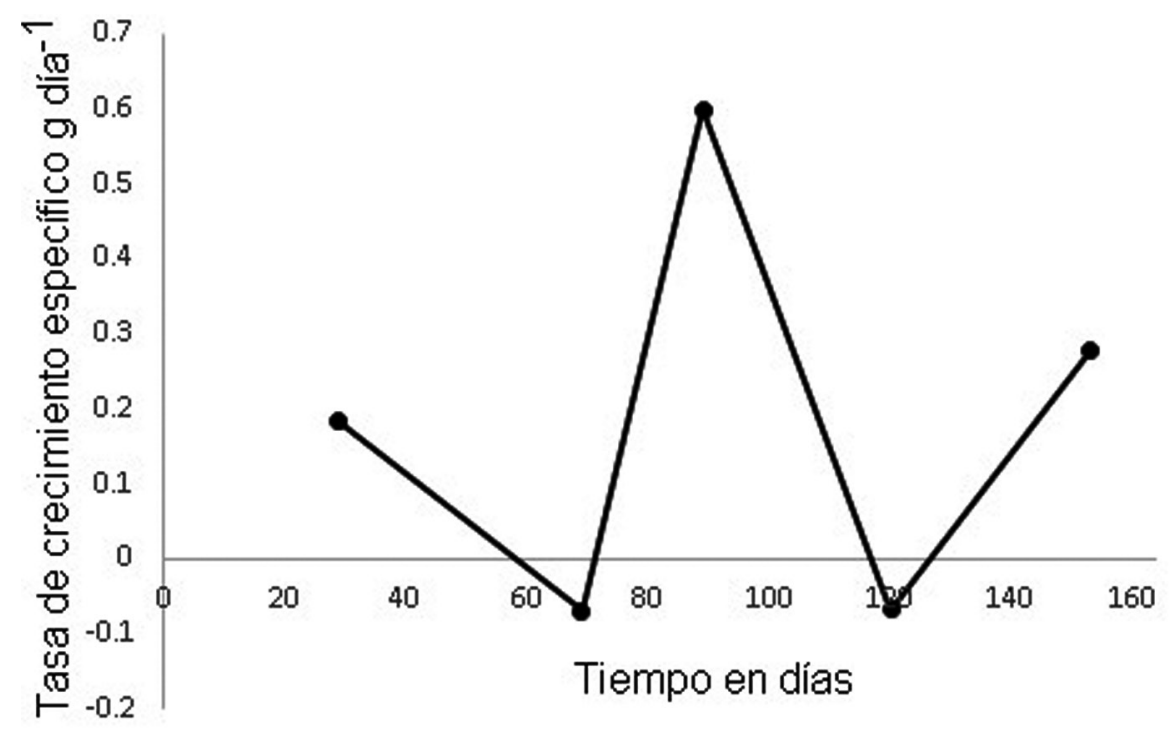

Figura No. 5. Tasa de crecimiento específico de la langosta (Panulirus argus), cultivada en jaulas flotantes en el Refugio de Vida Silvestre Cayos Perla, Nicaragua, del 21 de diciembre de 2018 al 23 de mayo de 2019.

De la ecuación 2, $t=t_{o}-\left\{\ln \left[1-\left(L_{t} / L_{\infty}\right)\right]\right\} / k$ y utilizando los valores de los parámetros del modelo de crecimiento de Von Bertalanffy, reportados por Borda y Cruz, 2006 y de León Gónzalez, 2005, el tiempo estimado que tardaría la langosta en crecer en el mismo intervalo de $L C$ observado en este estudio, bajo condiciones naturales, es de 122, 88 y 69 días, para el límite inferior, promedio y superior, de los parámetros del modelo, respectivamente, estos valores representan del 45 al $80 \%$ del tiempo observado en el estudio.

Sin embargo, al analizar las etapas de crecimiento identificadas (Figura No. 2), se observa que los primeros 69 días del cultivo, no se registraron cambios en el crecimiento, pero en los 84 días de la segunda etapa, se observó un incremento de $5.7 \mathrm{~mm}$ en la LC, con un tiempo teórico estimado de 116, 84 y 65 días, para el límite inferior, promedio y superior, de los parámetros del modelo, respectivamente, donde se percibe que el tiempo crecimiento en LC de ésta etapa es similar al promedio observado en la naturaleza.

Consideramos que existen dos factores que podrían explicar este comportamiento, por un lado esta la recuperación de las langostas después del estrés del translado, asociada a la primera etapa; por otro lado podría ser la diferencias de densidades de 10.6 y 6.3 organismos $\mathrm{m}^{3}$, durante los primeros 89 días del cultivo y los 64 días restantes, respectivamente; este comportamiento ha sido observado por Matthews \& Maxwell (2007), en cultivo de lanosta P. argus en laboratorio y Solanki y otros (2012), en cultivo de langosta P. polyphagus en jaulas flotantes en la bahía Sarkeshware, India.

Con relación a las características del agua durante el estudio, la temperatura fluctuó de 27.5 a $30{ }^{\circ} \mathrm{C}$, con un promedio de $28.5^{\circ} \mathrm{C}$; el pH osciló de 7.2 a 8.3 unidades, con un promedio de 7.6; la salinidad de 33.3 a $35 \mathrm{~g} \mathrm{~L}^{-1}$, con un promedio de $34.4 \mathrm{~g} \mathrm{~L}^{-1}$, la turbidez siempre fue mayor que un metro y la velocidad de la corriente fluctuó de 0.2 a $0.4 \mathrm{ft} \mathrm{s}^{-1}$. 


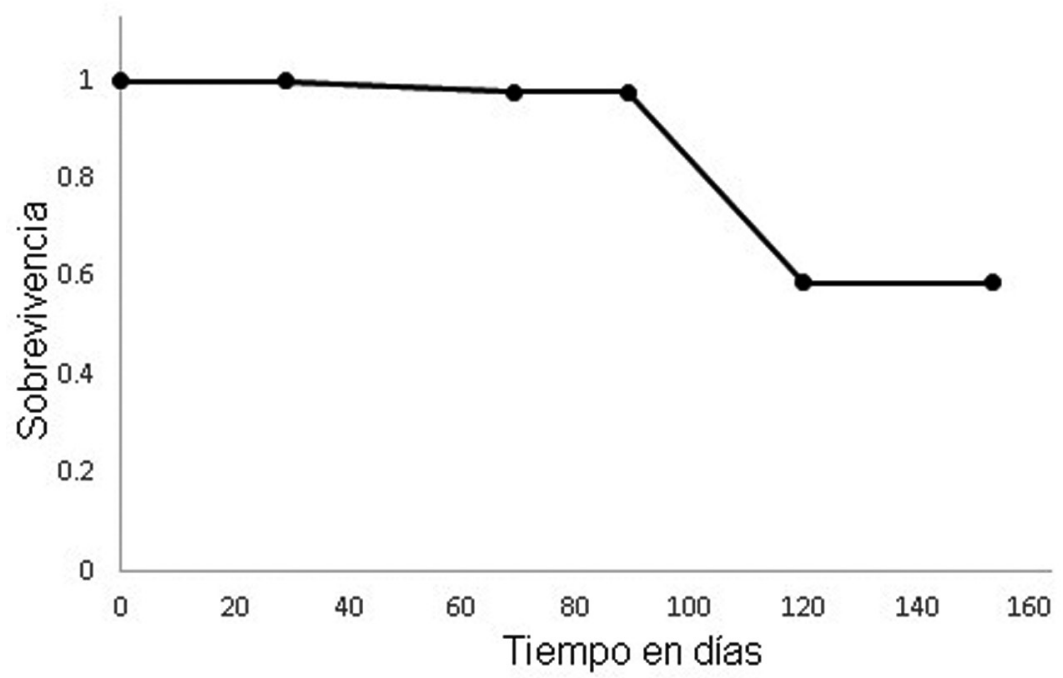

Figura No. 5. Sobrevivencia de la langosta (Panulirus argus), cultivada en jaulas flotantes en el refugio de vida silvestre Cayos Perla, Nicaragua, del 21 de diciembre de 2018 al 23 de mayo de 2019.

Durante los primeros 89 días del cultivo, la langosta presentó una alta sobrevivencia, aunque un crecimiento nulo; en el periodo de 90 a 120 días del cultivo, se observó un 39\% de mortalidad, asociado a la muda y probable canibalismo, así como al saqueo; ya en la fase final, durante el periodo de 120 a 153 días del cultivo, no se registró mortalidad (Figura No. 5) y se presentaron los mayores incrementos en peso y longitud de la langosta (Figuras No. 2, 3 y 4).

La relación entre el peso (W) y la longitud del cefalotórax (CL), para este estudio está dada por la ecuación (Figura No. 6):

$$
W=0.02788 L C^{2.2074}
$$

Donde $\mathrm{W}$ es el peso en gramos de la langosta y LC es la longitud del cefalotórax en $\mathrm{mm}$. Con intervalos de confianza al 95\% de los coeficientes, 0.0056 - 0.1168 y $1.8801-2.5735$, para 0.02788 y 2.2074 , respectivamente. Estas estimaciones difieren estadísticamente de los reportados por Alzugaray y Puga (2012), para el crecimiento de la langosta en la plataforma suroccidental de Cuba, $W=0.00243 L C^{2.764}$ y a las de Borda y Cruz (2006), para el archipiélago de San Andrés, Providencia y Santa Catalina, $W=0.002582$ LC ${ }^{2.7461}$. Estas diferencias se pueden deber a que el intervalo de las variables observado en este estudio es muy corto, comparado al utilizado para el ajuste de los modelos por los autores citados.

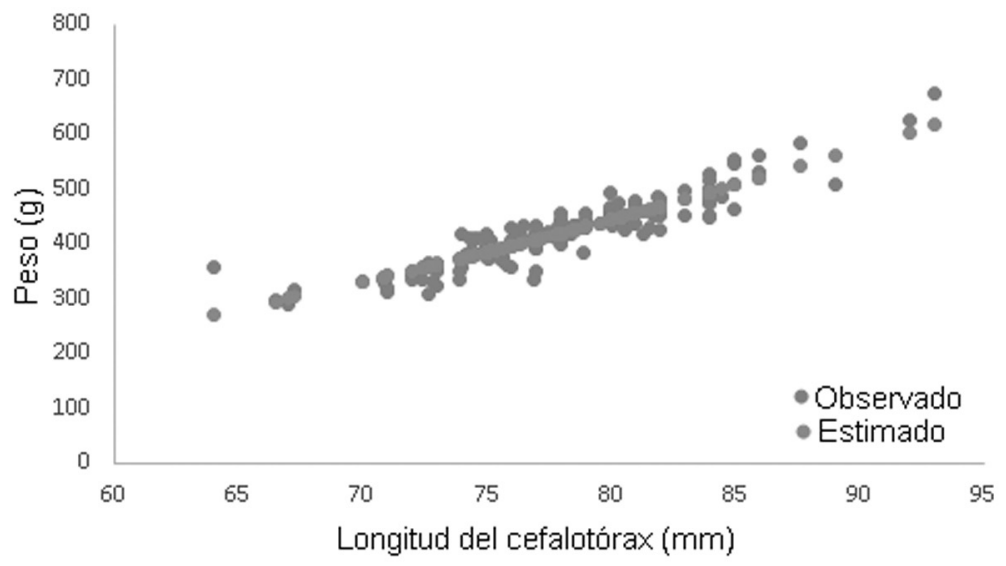

Figura No. 6. Relación peso vs longitud, de la langosta (Panulirus argus), cultivada en jaulas flotantes en el Refugio de Fauna Silvestre Cayos Perla, Nicaragua. 


\section{Conclusiones}

- Del cultivo de 85 langostas (Panulirus argus), en una jaula flotante de 2x2x2.30 m, en el Refugio de Vida Silvestre Cayos Perla, Nicaragua, del 28 de diciembre de 2018 al 23 de mayo de 2019, alimentadas con sardina a razón del 7\% de su peso, se desprenden las siguientes conclusiones:

- Las langostas incrementaron en promedio en 153 días de cultivo, $18.3 \mathrm{~mm}$ de longitud total (LT), 6.1 $\mathrm{mm}$ de longitud del cefalotórax y $93 \mathrm{~g}$ de peso. Se observaron valores negativos en la SGR, asociados a las mudas de la langosta. También se observaron valores positivos (o.18 -0.6), que manifiestan el potencial de crecimiento bajo condiciones de cultivo en jaulas flotantes.

- Se identificaron dos etapas de crecimiento, la primera con crecimiento nulo y con alta mortalidad al final, pero la segunda con crecimiento similar al observado en su estado natural y mortalidad nula.

- $\quad$ El agua presentó valores promedio de temperatura $28.5^{\circ} \mathrm{C}, \mathrm{pH}$ de 7.6 , salinidad $34.4 \mathrm{~g} \mathrm{~L}^{-1}$, turbidez mayor a un metro y velocidad de la corriente de $0.2 \mathrm{a} 0.4 \mathrm{ft} \mathrm{s}^{-1}$.

- La sobrevivencia presento valores de $98 \%$ al inicio del cultivo, después presentó un descenso brusco $(59 \%)$, para concluir con $100 \%$ en las últimas dos biometrías.

- La relación entre el peso (W) y la longitud del cefalotórax (CL), para este estudio está dada por la ecuación:

$$
W=0.02788 L C^{2.2074}
$$

- De los resultados de este estudio, se pude decir que el cultivo de la langosta (Panulirus argus) en jaulas flotantes en la región del Refugio de Vida Silvestre Cayos Perla, Nicaragua, tiene un alto potencial.

- Se sugiere hacer investigaciones para evaluar el impacto del traslado de las langostas de su hábitat natural hacia las jaulas para su cultivo; así como las densidades óptimas por jaula.

\section{Agradecimiento}

Esta publicación obtuvo el financiamiento del Proyecto Ecosistemas Costeros, auspiciado por: Unión Europea, Dka Austria, Cooperación Austriaca para el Desarrollo y Horizontzooo.

\section{Lista de referencias}

Alzugaray Martínez, R., y Puga Millán, R. (Diciembre de 2012). Comparación entre dos modelos estructurados por edades, aplicados a la pesquería de langosta, Panulirus argus (Latreille, 1804), en la región suroriental de Cuba. Rev. Mar. Cost., 4, 131-143. doi:http://dx.doi.org/10.15359/revmar.4.9

Borda, C. A., \& Cruz, R. (2006). Evaluación de la pesquería de Langosta (Panulirus argus) en el archipiélago de San András, Provincia y Santa Catalina, Caribe Colombiano. Rev. Invest. Mar, 3(27), 219-230.

de León Gónzalez, M. E. (2005). Variabilidad temporal de los parámetros poblacionales de la langosta espinosa del Caribe Panulirus argus (Latreille, 1804) en aguas de Cuba. La Paz, Baja California Sur, México: Centro de Investigaciones Biológicas del Noroeste, S.C.

Ehrhardt, N. M. (2005). Population dynamic characteristics and sustainability mechanisms in key western central atlantic spiny lobster, Panulirus argus, fisheries. Bulletin of marine science, 76(2), 501-525. 
FAO (2003). Segundo Taller sobre Manejo de las Pesquerías de la Langosta Espinosa del Área de la Copaco. FAO. La Habana, Cuba: FAO.

Jeffs, A. (2010). Status and Challengers for advancing lobster aquaculture. J. Mar. Biol. Ass. India, 52(2), 320-326.

Jeffs, A., y Davis, M. (2003). An Assessment of the Aquaculture Potential of the Caribbean Spiny Lobster, Panulirus argus. 54th Gulf and Caribbean Fisheries Institute(54), 413-426.

Matthews, T. R., \& Maxwell, K. E. (2007). Growth and Mortality of Captive Caribbean Spiny Lobsters, Panulirus argus, in Florida, USA. 58th Gulf and Caribbean Fisheries Institute, 358-366.

Ríos Lara, G. V., Cervera Cervera, K., Espinoza Méndez, J. C., Pérez Pérez, M., Zetina Moguel, C., \& Chablé Ek, F. (1998). Estimación de las densidades de Langosta Espinosa (Panulirus argus) y Caracol Rosado (Strombus gigas) en el Area Central del Arrecife Alacranes, Yucatan, Mexico. Proceedings of the 5oth Gulf and Caribbean Fisheries Institute, 104-127.

Rivera Menéses, R., Suárez Sánchez, J., Pérez González, L. d., Van der Wal, J. C., Muñoz Nava, H., \& Morales Moreno, S. (June de 2013). Comparison of experimentally elaborated food from regionally available products with commercial foods of common carp (Cyprinus carpio) in the Mexican highlands. International Journal of Fisheries and Aquaculture, 5(6), 136-141. doi:10.5897/IJFA12.060

Solanki, Y., Jetani, K. L., Khan, S. I., Nayan, P., Makawana, N. P., \& Rather, M. A. (2012). Effect of stocking density on growth and survival rate of spiny lobster (Panulirus polyphagus) in cage culture system. International Journal of Aquatic Science, 3(1), 3-14. 